\title{
La neoplasia intraepitelial del cérvix en el Centro Médico de los Andes: Diagnóstico, tratamiento y seguimiento
}

\author{
Oscar Hernando Arismendy Sanabria*
}

\section{Introducción}

Prevalencia. Toda vez que es ésta, una patología esencialmente asintomática, su diagnóstico correcto sólo puede basarse en la realización de biopsias dirigidas, efectuadas en una muestra significativa de la población estudiada. Dados los costos y la invasividad de tal metodología diagnóstica, este tipo de proceso no se ha conducido en ningún trabajo de los revisados, y las estadísticas son en realidad tan sólo aproximaciones numéricas al problema.

Mencionaré por tanto tan sólo un trabajo de 1984, el cual establece el diagnóstico de NIC sobre bases citológicas. Según este informe que utiliza como población 796.337 mujeres que se sometieron a toma de citología en los Estados Unidos en 1981, la incidencia global sería de $2,32 \%$. El análisis de la distribución etárea muestra una prevalencia del $1 \%$ en niñas entre los 10 y 14 años, con un incremento progresivo hasta $2,8 \%$ en el grupo de 20 a 24 años, para alcanzar nuevamente un valor de $1,08 \%$ entre los 50 y 54 años, y seguir bajando hasta el $0,97 \%$ entre los 60 y 64 años, donde desarrolla una meseta con discreta tendencia al descenso. Este mismo informe concluye que el promedio de edad para el desarrollo de la enfermedad es de 25 años; pero si se la divide por grados, el promedio de edad para el NIC 3 sube a los 28 años (con una prevalencia mayor en el grupo entre 30 y 34 años: $5,5 \%$ ). Se observa pues que la neoplasia intraepitelial del cérvix es una enfermedad de mujeres jóvenes, y llama la atención la prevalencia notoria en el grupo de púberes y adolescentes (2).

\section{Conducta sexual femenina}

Desde hace aproximadamente un siglo, los estudios realizados han mostrado que la conducta sexual desempeña un papel importante en el origen del cáncer cervical y de sus precursores. Se ha señalado en varias encuestas epidemiológicas a las siguientes características como los factores asociados de mayor significancia en la población afectada por NIC: edad temprana al momento del primer coito, corta edad al momento del primer embarazo, gran multiparidad, y un intervalo intergenésico corto. Otros factores menos importantes pero igualmente mencionados por la década de los sesenta fueron: la promiscuidad sexual y la existencia de enfermedades venéreas $(1-5,10,41)$.

\footnotetext{
* Residente I Ginecología y Obstetricia. Hospital Universitario San Ignacio.
}

\section{Factor masculino}

En los años setenta se dio una gran importancia al componente masculino e incluso se acuñó el término de «varón de alto riesgo", refiriéndose a la mayor incidencia de neoplasia cervical en las compañeras sexuales de hombres con Ca de próstata y con Ca de pene; y en la segunda y tercera esposas de un hombre cuya primera compañera hubiera sufrido de la enfermedad $(1,3,5,10)$.

\section{Raza}

En los Estados Unidos se reportaban incidencias más elevadas de NIC en la población negra, lo que los llevó a pensar en diferencias raciales de susceptibilidad a la enfermedad. Igual sucedió con la observación de menores frecuencias de neoplasia cervical en los judíos. Estudios epidemiológicos controlados mostraron que tales diferencias eran función realmente de covariables como el nivel socioeconómico y las costumbres sexuales $(1,3,5)$.

\section{Clase socioeconómica}

Estudios tan antiguos como los de Vineberg en 1906 y más recientes como los de Wakefield de 1973 han demostrado una incidencia incrementada de la enfermedad cervical neoplásica de hasta tres veces en las clases socioeconómicas bajas. Un estudio de Skegg en 1982 pareció refutar esta teoría argumentando que la conducta sexual promiscua de sus estudiados podía explicar los resultados. Persiste aún la controversia al respecto $(1,3-5,8,10)$.

\section{Métodos anticonceptivos}

Los estudios tradicionales no han encontrado diferencias estadísticamente significativas en la incidencia del NIC en las usuarias de anticonceptivos orales, dispositivos intrauterinos o diafragmas. Vessey en 1983 en cambio reportó una mayor incidencia de NIC en usuarias de anticonceptivos orales, con una clara relación con la duración del uso. Se necesitan estudios mayores para sustentar este hallazgo $(1,3$, 5, 9-10).

\section{Químicos exógenos}

Existen algunos estudios que relacionan el hábito de fumar con la posibilidad de desarrollar NIC. Se controvierte en la mayoría de revisiones la metodología de los mismos ( 1 , 9-10). 


\section{Etiopatogenia del NIC}

Toda vez que actualmente el NIC se considera como un estadio dentro de una serie de cambios que terminan en el desarrollo del cáncer cervical, se mencionarán aquí aquellos hechos que tienen que ver con el proceso del origen del cáncer en tal localización: la célula blanco (en este caso el epitelio de la zona de transformación), el agente carcinógeno y el proceso mismo de la transformación neoplásica.

\section{Zona de transformación}

Se describe inicialmente la zona de transformación histológicamente, y se mencionan luego los cambios metaplásicos, considerados como normales dentro de la zona de transformación típica.

\section{Histología de la zona de transformación}

I.a superficie de la portio vaginalis del cérvix está cubierta por epitelio estratificado no queratinizado semejante y que establece continuidad en el epitelio vaginal, mientras que el canal endocervical está cubierto por epitelio columnar diferente del epitelio endometrial. Las descripciones clásicas establecían la transición entre uno y otro tipo de epitelio como una zona de transición abrupta denominada la «unión escamocolumnar». En realidad el paso de uno a otro epitelio se hace en una zona más o menos amplia desde el punto de vista histológico; es a esta área a la que se ha denominado «zona de transformación». Su extensión varía de 1 a $10 \mathrm{mms}$., no solamente entre uno y otro individuo sino en el mismo individuo. Su localización tampoco es rígida: coincide con el orificio cervical externo anatómico con frecuencia; pero en la mujer menopáusica puede adentrarse en el canal endocervical; en la embarazada, en la mayoría de mujeres menstruantes, en las usuarias de anticonceptivos orales, en la pubertad y en cualquier circunstancia en que se utilicen altas dosis de estrógenos se puede encontrar en el exocérvix (el mal llamado ectropión). (1, 3, 5, 29).

\section{Metaplasia escamosa}

La unión entre los epitelios columnar y escamoso originales es transitoria. Presumiblemente como resultado de la exposición a altas concentraciones plasmáticas de estrógenos $\mathrm{y}$ a las condiciones de ph ácido vaginal, el epitelio columnar va siendo reemplazado por epitelio escamoso metaplásico, convirtiendo así la unión escamocolumnar inicial en una unión escamo-escamosa entre el epitelio escamoso plano original y el metaplásico. El proceso metaplásico es inicialmente aislado pero puede al hacerse multifocal, coalescer y reemplazar toda la unión escamocolumnar $(3,5,29)$.

La metaplasia del epitelio columnar ocurre durante toda la vida femenina, pero es más activa en tres fases de la misma: el período perinatal, la menarquia y el primer embarazo. Los períodos de mayor actividad metaplásica coinciden con aquellos en que los niveles estrogénicos son mayores; y son precisamente esos momentos los más riesgosos para el desarrollo de cambios neoplásicos, toda vez que en ellos la rapidez de la metaplasia conlleva un cierto grado de inmadurez del epitelio formado (29).

En los cambios inflamatorios es importante describir la presencia de células inflamatorias que representarían algún grado de defensa inmune del huésped contra las células epiteliales transformadas; de tal manera podría inferirse que la progresión hasta estadios avanzados de la enfermedad (v.g. NIC III o Ca invasivo), se presentaría como consecuencia de fallas en el sistema de vigilancia inmunológica y la regresión de la misma en los estados precoces (v.g. NIC I) como un éxito del mismo (5).

\section{Carcinógenos y neoplasia cervical}

Dado que los factores asociados con un alto riesgo de neoplasia cervical se relacionan con eventos sexuales, y dado que la enfermedad no se encuentra prácticamente en mujeres vírgenes, se han establecido estudios en dos líneas de posibles agentes causales: microorganismos sexualmente transmitidos en un lado, y factores inherentes al semen por el otro.

\section{Teoría de los microorganismos carcinogénicos}

Los estudios realizados han relacionado básicamente a cuatro microorganismos: el Citomegalovirus (CMV), el herpesvirus tipo II (HSV-2), el papilomavirus (HPV), y la Clamydia (1, 5-7, 11, 14-17). En los reportes más recientes el HPV lleva la delantera como implicado, pero para todos ellos se han encontrado las siguientes evidencias sugestivas:

1. Recuperación de antígenos de los microorganismos en cuellos afectos por lesiones neoplásicas (con mayor frecuencia en las lesiones tipo NIC que en el cáncer invasivo) en mayor proporción que en los cuellos sanos.

2. Demostración de mayores porcentajes de positividad de anticuerpos séricos en los pacientes con NIC o Ca invasor frente a grupos de control.

3. Demostración de oncogenicidad in vitro al menos para los virus estudiados.

\section{Teorías sobre la carcinogénesis del semen}

Algunos investigadores como Reid y Coppleson favorecen la teoría del papel masculino sobre los microorganismos en la génesis de la neoplasia del cuello uterino. Ellos sugieren como carcinógeno al DNA del espermatozoide, dado que puede ser incorporado en la información nucleica de las células epiteliales normales o metaplásicas en un patrón similar al de los virus carcinogénicos. En el esperma hay dos proteínas altamente básicas: protamina e histona. La concentración relativa de las mismas determina la afinidad de reacción cruzada con el DNA de la célula huésped y con los microfilamentos de superficie, en forma lineal (por lo menos in vitro) $(1,5,10)$.

\section{Transformación neoplásica}

El hecho que partículas diferentes puedan suscitar la misma respuesta por parte del epitelio, no permite escoger entre ellas a un solo agente carcinógeno común y ha llevado a dos posibilidades: la primera sería aceptar que es la combinación de varias partículas como cocarcinógenos la responsable en la producción de la lesión (incluso con la factibilidad que el impacto de una de ellas pudiera no ser identificado en la actualidad por los métodos técnicos disponibles -efecto «hit and run»-), y la segunda en la que se relegaría a las partículas ya estudiadas al papel de agentes carcinógenos, dejando por establecer aún la naturaleza del carcinógeno real (5). 
Cualquiera que sea la realidad, una vez sometida al «agente agresor», la célula columnar pierde su actividad secretoria, se redondea, presenta alteraciones en el sistema de filamentos internos (citoesqueleto), y aparecen numerosas proyecciones filamentarias en su superficie; cambios estos que la caracterizan como una célula capaz de sintetizar queratina. Luego las células entran en una fase de crecimiento y multiplicación que responde por la formación de varias capas de células. Tanto la fase de reorganización del citoesqueleto, como la fase posterior de mitosis son consideradas como momentos de mayor susceptibilidad de transformación neoplásica $(1,5)$.

Los cambios morfológicos celulares pueden por razones físicas ocluir la salida de proteínas altamente especializadas involucradas en el control del desarrollo celular como es el caso de la 1, antitripsina (descrita como disminuida en el NIC por MilfordWard en 1984) (5).

\section{Material y métodos}

Se revisaron los informes de patología del C.M.A.; se localizaron los resultados positivos para NIC en biopsias y en cono diagnóstico.

Una vez localizados se revisaron las historias clínicas de tales pacientes y se procedió a diligenciar una tabla de datos precodificados por computador con información referente a la metodología diagnóstica, terapéutica y de seguimiento de los mismos.

Dado que uno de los objetivos del trabajo era evaluar la correlación entre los sistemas diagnósticos empleados, resultaba obvia la necesidad de incluir controles, para la tabulación adecuada de los resultados.

Como control para la correlación entre citología y patología se usaron pacientes seleccionados al azar en los archivos de patología, que hubieran sido sometidas a histerectomías por patologías benignas.

El control para el estudio colposcopia-patología se constituyó con aquellas pacientes que fueron estudiadas por colposcopia por sospecha de patología cervical benigna y en quienes se tomó muestra por biopsia dirigida.

Para la identificación de las pacientes se empleó la categorización por médico tratante unida a un listado alfabético accesorio.

\section{Metodología diagnóstica}

En la implementación del diagnóstico se revisaron cuatro opciones a saber:

1. Citología: se clasificó en I, II, III, IV, de acuerdo con el resultado del Pap. Se incluyó una subcategoría, llamada IIA, en la cual se incluyeron aquellas citologías que aunque correspondían a Pap II, tenían aclaración respecto a la presencia de atipias entre moderadas y severas, las cuales corresponden según el departamento de patología a atipias que no pueden explicarse sobre la base de influencias inflamatorias. Una vez así divididas se clasificaron en dos grupos:

- Positivas: si eran Pap IIA, III ó IV.

- Negativas: si eran Pap I ó II.

2. Colposcopia: se dividió en dos categorías: positiva o negativa para NIC.
- Positiva: incluyó hallazgo de base, mosaico, epitelio acetoblanco, y leucoplasia.

- Negativa: si los hallazgos eran compatibles con zona de transformación típica (incluyendo la metaplasia escamosa), o con cambios inflamatorios. Si eran colposcópicamente diagnósticos de carcinoma invasor eran excluidas de la tabulación.

3. Patología: dado que el departamento de patología del C.M.A. usa la terminología Displasia-Carcinoma in situ, y el presente trabajo se basa en la nomenclatura de Richart, se reclasificaron los resultados de patología como ha sido descrito en el texto en:

- NIC 1 para la displasia leve.

- NIC 2 para la displasia moderada.

- NIC 3 para la displasia severa y el carcinoma in situ.

4. Positividad en patología para papilomavirus: se consideraron en dos grupos:

- Positivo: si el diagnóstico de lesión por HPV estaba confirmado además de las características histológicas, por reacciones de inmunohistoquímica.

- Negativo: si no existía tal confirmación.

\section{Terapéutica empleada}

Se utilizaron las siguientes categorías, que son las disponibles y empleadas en el C.M.A. para el manejo de estos casos:

1. Manejo expectante: si la paciente había sido sometida a control citológico periódico una vez establecido el diagnóstico, independientemente si recibieron o no tratamiento antiinflamatorio tópico de alguna naturaleza.

2. Crioterapia.

3. Cauterización.

4. Cono terapéutico.

5. Histerectomía total.

6. Histerectomía ampliada.

Una vez analizados los métodos individuales, se clasificaron de acuerdo con el texto en:

- Manejo expectante.

- Métodos conservativos

- Métodos radicales.

\section{Metodología del seguimiento}

Inicialmente se había categorizado en dos grupos según las pacientes que hubieran sido controladas por medio de citología o colposcopia, pero en vista que sólo se encontraron 6 pacientes que tenían controles por el segundo método, y que los resultados del mismo no habían influido en el manejo de las mismas se excluyó tal información del trabajo.

El control de citología dividió a las pacientes en:

1. Pacientes con seguimiento: si tenían citología de control en los primeros 6 meses del tratamiento con resultado Pap Ió II, y además tenían los mismos resultados en citologías tomadas cuando menos en los últimos 12 meses.

2. Pacientes sin seguimiento: se dividieron en 2 subgrupos:

- Pacientes recientes: si la paciente había sido sometida a tratamiento en los últimos 6 meses y por lo tanto no se esperaba que tuviese citologías de control.

- Pacientes perdidas: si la paciente había sido sometida a tratamiento hace más de 6 meses, y no tenían ningún control 
citológico; o si habiendo tenido un control citológico inicial, no tenía uno reciente (de menos de 1 año).

\section{Resultados del seguimiento}

Dado que el más antiguo de los casos apenas supera los tres años, no se hizo ninguna categorización de pacientes curadas; y dado que sólo se trató de 5 casos, simplemente se presentará un breve resumen de las historias de los mismos.

\section{Resultados}

La revisión comprende los archivos existentes desde la fundación del C.M.A. hasta el 30 de mayo de 1986.

Para la correlación de Citología vs. Patología se utilizaron los 105 pacientes encontrados en los archivos en el lapso estudiado con Dx de NIC y un control de 65 pacientes. Los resultados se muestran en la tabla 1. Rápidamente se puede ver que de los pacientes con NIC (105), el 21,76\% corresponden a NIC 2, y la mitad de los restantes a NIC 1 ó 3 (aprox. el $37 \%$ para cada uno). En el grupo control el $57 \%$ tiene diagnóstico de cervicitis y el $43 \%$ de tejido cervical normal.

Del total de 170 pacientes, hubo 15 pacientes cuyas historias clínicas no pudieron encontrarse; por lo tanto al ignorarse el resultado de sus citologías las tablas de correlación entre citología y patología debieron elaborarse con 155 pacientes: 90 pacientes con NIC vs. 65 con Dx no NIC.

En la tabla 2 se aprecia el cruce entre los no NIC y los diferentes grados de NIC con los resultados de citología. Se observa rápidamente una tasa de falsos positivos para la citología del 17,30\%, y de falsos negativos del 8,16\% (todos correspondientes a NIC I).

Con estos mismos resultados se confeccionó la tabla 3 para el cálculo de la significancia estadística de la citología en la predicción del NIC. Los resultados de significancia fueron:
Valor predictivo positivo:
$82,69 \%$
Valor predictivo negativo:
$91,83 \%$
Sensibilidad:
$95,55 \%$
Especificidad:
$71,42 \%$

La correlación entre colposcopia y diagnóstico de patología se efectuó con base en 63 pacientes (Ver tabla 4). La tasa de resultante de falsos positivos es del $17,5 \%$ y la de falsos negativos es de $69,56 \%$. Con estos datos se calculó la significancia estadística de la colposcopia en el C.M.A. para el diagnóstico de NIC con los siguientes resultados:

$\begin{array}{ll}\text { Valor predictivo positivo: } & 82,50 \% \\ \text { Valor predictivo negativo: } & 30,43 \% \\ \text { Sensibilidad: } & 67,34 \%\end{array}$

Especificidad: $\quad 50,00 \%$

La positividad para infección por HPV según los criterios establecidos sólo se encontró en 8 de los 105 casos revisados $(8,16 \%)$, distribuida en 4 casos de NIC 1,3 casos de NIC 2 , y 1 caso con NIC 3 . El diagnóstico se hizo por citología en 2 casos de los revisados, y por colposcopia en ninguno.

La evaluación del tratamiento sólo se logró en 71 pacientes por imposibilidad de encontrar ciertas historias clínicas (32 pacientes), o porque la paciente no recibió tratamiento por no volver a consulta médica (2 pacientes).

En orden de frecuencia los sistemas de tratamiento empleados en el C.M.A. son: crioterapia en 25 pacientes $(35,21 \%)$; manejo expectante en $20(28,17 \%)$; cono en 12
(16,9\%); histerectomía en $10(14,08 \%)$; histerectomía ampliada en $3(4,22 \%)$; y cauterización en $1(1,4 \%)$.

Si se utiliza la clasificación descrita en el marco conceptual, los métodos radicales, los conservativos y el manejo expectante se usaron casi con la misma frecuencia $(35,2 \%$, $36,61 \%$, y $28,17 \%$ ).

Al subdividirlas de acuerdo al grado de NIC se observa:

1. Que la mitad de las pacientes con NIC 1 fueron tratadas en forma expectante y la otra mitad con métodos conservativos. Figura un caso tratado con cono, que en realidad corresponde a una paciente quien se presentó con una citología IIA después de una histerectomía subtotal, y fue sometida a una amputación del cuello con el resultado histopatológico descrito (NIC 1).

2. Que casi la mitad de las pacientes con NIC $2(47,37 \%)$ fueron sometidas a manejo expectante, en tanto que una tercera parte de ellas fueron manejadas con métodos conservativos $(31,57 \%)$, y una quinta parte con métodos radicales $(21,05 \%)$.

3. Que más de las tres cuartas partes $(76,91 \%)$ de las pacientes con NIC 3 fueron sometidas a métodos radicales de manejo, en tanto que una quinta parte fueron sometidas a manejo conservativo (19,23\%), y tan sólo se les hizo manejo expectante al 3,84\% de ellas. (Ver tabla 5).

Con respecto a la metodología del seguimiento se encontraron 55 de 71 pacientes con seguimiento, y 16 de 71 pacientes sin seguimiento, (de las cuales 6 fueron pacientes recientes y 11 pacientes perdidas).

Es decir que en cuanto al resultado del seguimiento sólo se analizan 55 pacientes. En ellas se encontraron 3 pacientes tratadas con crioterapia y 2 pacientes con cono, que habían presentado evidencia de recidiva o progreso de la enfermedad (evaluada por citología anormal luego de más de 6 meses de haber sido tratada), para una tasa de fallas del tratamiento del $9,09 \%$, que corresponderían además a una falla del $16,66 \%$ de los conos terapéuticos y del $12 \%$ de las crioterapias.

Vale la pena mencionar que una de las pacientes con recidiva por crioterapia se encuentra en tratamiento por leucemia con busulfan (caso en el cual la inmunosupresión explicaría la recidiva), lo cual daría una tasa de falla corregida para la crioterapia del $8 \%$.

Los pacientes con cono que presentaron falla del tratamiento son:

- Paciente con citología IV, a quien se le practicó un cono diagnóstico que mostró un NIC II con vértices del cono libres de lesión; se presentó a control con una citología Pap IV, por lo cual se llevó a histerectomía (el espécimen mostró un NIC II); los controles citológicos recientes de cúpula son Pap I.

- Paciente con citología III, se manejó con cono el cual mostró un NIC III con vértice del cono libre; el control citológico fue Pap III a los 6 meses del tratamiento; se sometió a histerectomía total; el control citológico fue Pap III, por lo cual se llevó a colposcopia con biopsias dirigidas que mostraron neoplasia intraepitelial de vagina que se manejó con crioterapia; los controles posteriores son Pap I.

Este análisis permite ver que sólo se presentó falla real en un cono para una tasa corregida de recidiva del $8,33 \%$, para este método y una tasa corregida total de recidiva del 5,45\%.

Existe además una paciente que mencionaré como caso interesante aunque no modifica las estadísticas por tratarse de un cono diagnóstico: 
- Paciente quien fue sometida a un cono por una citología Pap III; el espécimen fue reportado (fuera del C.M.A.), como cervicitis aguda y crónica. La paciente presenta ya en el C.M.A. una citología Pap III, se le practica un cono diagnóstico que muestra un carcinoma infiltrante IIb por lo cual es sometida a radioterapia.

Tabla 1

TABLA DE ANALISIS DE LOS RESULTADOS DE PATOLOGIA

\begin{tabular}{|lcccc|}
\hline & NIC 1 & NIC 2 & NIC 3 & Total \\
\hline NIC \#: & 40 & 26 & 39 & 105 \\
NIC \%: & $30,09 \%$ & $24,76 \%$ & $37,14 \%$ & $100,00 \%$ \\
\hline
\end{tabular}

\begin{tabular}{|lccc|}
\hline & Cervicitis & Normal & Total \\
\hline No NIC \#: & 37 & 28 & 65 \\
No NIC \%: & $56,92 \%$ & $43,08 \%$ & $100,00 \%$ \\
\hline
\end{tabular}

Tabla 2

RESULTADOS DE LA CORRELACION ENTRE CITOLOGIAS Y PATOLOGIAS

\begin{tabular}{|lcccccccc|}
\hline & \multicolumn{2}{c}{ NIC 1 } & \multicolumn{2}{c|}{ NIC 2 } & \multicolumn{2}{c|}{ NIC 3 } & \multicolumn{2}{c|}{ NO NIC } \\
\hline & $\%$ & $\#$ & $\%$ & $\#$ & $\%$ & $\#$ & $\%$ & $\#$ \\
Negativa: & 8,16 & 4 & 0 & 0 & 0 & 0 & 91,83 & 45 \\
Positiva: & 30,70 & 32 & 22,10 & 23 & 29,81 & 31 & 17,30 & 18 \\
Total: & 23,53 & 36 & 15,03 & 23 & 20,26 & 31 & 41,17 & 65 \\
\hline
\end{tabular}

Tabla 3

RESULTADOS TOTALES DE CORRELACION ENTRE PATOLOGIA Y CITOLOGIA

\begin{tabular}{|llrlrc|}
\hline & \multicolumn{1}{c}{ NIC } & \multicolumn{3}{c|}{ NO NIC } & TOTAL \\
\hline Negativa & $8,16 \%$ & 4 & $91,83 \%$ & 45 & 49 \\
Positiva & $82,69 \%$ & 86 & $17,30 \%$ & 18 & 104 \\
Total & $58,8 \%$ & 90 & $41,17 \%$ & 63 & 153 \\
& $\%$ & No. & $\%$ & No. & No. \\
\hline
\end{tabular}

Tabla 4

RESULTADOS DE LA CORRELACION ENTRE COLPOSCOPIA Y PATOLOGIA

\begin{tabular}{|llllrc|}
\hline & \multicolumn{2}{c}{ NIC } & \multicolumn{2}{c|}{ NO NIC } & TOTAL \\
\hline Positiva & $82,50 \%$ & 33 & $17,50 \%$ & 7 & 40 \\
Negativa & $69,56 \%$ & 16 & $30,43 \%$ & 7 & 23 \\
Total & $77,77 \%$ & 49 & $22,22 \%$ & 14 & 63 \\
& $\%$ & No. & $\%$ & No. & No. \\
\hline
\end{tabular}

Tabla 5

CLASIFICACION DE LOS TRATAMIENTOS EFECTUADOS DE ACUERDO CON EL DIAGNOSTICO PATOLOGICO

\begin{tabular}{|lcccccccc|}
\hline & \multicolumn{2}{l}{ NIC 1 } & \multicolumn{2}{l}{ NIC 2 } & \multicolumn{2}{l|}{ NIC 3 } & \multicolumn{2}{l|}{ TOTAL } \\
\hline Expectante: & 13 & $50,0 \%$ & 6 & $31,6 \%$ & 1 & $3,8 \%$ & 20 & $28,1 \%$ \\
Conservativo & 12 & $46,1 \%$ & 9 & $47,4 \%$ & 5 & $19,2 \%$ & 26 & \\
$36,6 \%$ & & & & & & & & \\
Radical: & 1 & $3,8 \%$ & 4 & $21,0 \%$ & $2076,9 \%$ & 25 & $35,2 \%$ \\
Total: & 26 & $36,1 \%$ & 19 & $26,7 \%$ & $2636,1 \%$ & 72 & $100 \%$ \\
& No & $\%$ & No & $\%$ & No\% & No & $\%$ \\
& & & & & & & \\
\hline
\end{tabular}

\section{Discusión}

La Neoplasia Intraepitelial del Cérvix, no es una patología de diagnóstico infrecuente en el C.M.A. Sinembargo el hecho que la mayoría de pacientes $(81,7 \%)$ sean sometidas a manejos ambulatorios, sumado a defectos en el proceso de registro de la información dificulta grandemente la recolección de datos referentes a la misma. En el presente estudio se aprecia cómo de 105 diagnósticos de patología positivos para la entidad, sólo se pudieron estudiar por este motivo el $83,81 \%$ en las correlaciones con citología; en tanto que en la evaluación del tratamiento, las fallas de registro de los nombres, especialmente, impidió la evaluación de un $30 \%$ de los casos localizados.

El análisis de los resultados referentes a citología vs. patología, es muy favorable, si se tiene en cuenta que Richart y Vailant en 1965 hablaban en el caso de displasias de falsos negativos del 28\%; en tanto que Coppleson y Brown en 1975 hablaban de un 40 a $20 \%$ según se incrementaba el grado de NIC (41). El estudio realizado habla el 8,16\%. La evaluación estadística: altísima sensibilidad con alta especificidad supone una correcta identificación de la población enferma, con un mínimo sobrediagnóstico, y hablan de una buena calidad en la recolección y evaluación de las muestras.

Cuando en cambio se revisa la colposcopia, los resultados son un tanto inquietantes. Existiendo la disponibilidad casi absoluta del aparato, y teniendo en cuenta que todos los autores revisados hablan de que la combinación de colposcopia y citología incrementa la certeza diagnóstica, tan sólo un $46 \%$ de los pacientes vistos son sometidos a los dos procedimientos antes de ir a tratamiento.

El análisis comparativo de los resultados estadísticos habla de falsos negativos del orden del $69,56 \%$. Aunque la colposcopia no es la panacea en el diagnóstico de las displasias o del Ca in situ, carrera (43) habla de una tasa de falsos negativos de apenas el $22,8 \%$.

La evaluación estadística revela una sensibilidad aceptable pero una especificidad mínima, resultados estos que inhabilitan desde el punto de vista matemático el uso de tal sistema diagnóstico de la institución. Muchos factores podrían dar cuenta de estos hechos: insuficiente entrenamiento en imagenología colposcópica, selección inadecuada del sitio de toma de las biopsias, realización de un sólo set de biopsias, por mencionar los menos elaborados.

La incidencia de la infección por papilomavirus fue mínima $(7,6 \%)$. La bibliografía consultada habla de la citología como el sistema ideal de detección de la misma. Este estudio muestra que el diagnóstico se sospechó mediante esta aproximación diagnóstica sólo en el $25 \%$ de los casos. Las explicaciones más probables son: o que la casuística es demasiado bajo, o que no se está familiarizado con sus características. En cambio, el hecho que ninguna de las pacientes se haya diagnosticado por colposcopia no sorprende, ya que se habla que salvo el condiloma florido no existe nada típico en la apariencia de estas infecciones y sí mucho en común con el NIC.

El manejo como era de esperarse tiende a ser más radical en la medida que se progresa en la severidad de la lesión. Si se aceptan los criterios de Sharp descritos en el texto, para el manejo del NIC con histerectomía, una proporción del 14\% (lo encontrado en el trabajo) resulta alta; empero, si se reconoce, como también se menciona, la continuidad del 
NIC al cáncer en una gran proporción de los casos, el uso de tal sistema terapéutico no resulta de ningún modo discutible.

La correlación de los diagnósticos de patología entre las biopsias y las piezas de histerectomía siempre confirmaron la presencia de la lesión aunque algunas veces en diferente grado (35\%). Cuando falló, el $90 \%$ de los casos fue por exceso en el grado adjudicado en la biopsia, y el $10 \%$ restante por defecto.

Que tan sólo un $15 \%$ de los pacientes susceptibles de tratamiento hallan desertado el seguimiento, confirman el supuesto planteado en la introducción sobre la facilidad de concientización del grueso de las pacientes manejadas en la institución respecto de su patología.

Comentar las tasas de fallas, requiere mayores volúmenes que los utilizados en esta revisión, aunque no deja de preocupar la falla de 1 de 12 conos realizados.

Obviamente se requieren proyectar estudios a más largo plazo para confirmar los resultados hasta aquí comentados, y espero que el instrumento de registro propuesto (Ver anexo 1), ayude a tal efecto.

Nota: Este anexo ha sido diseñado para incluir la patología maligna cervical, pero no está aún sistematizado.

\section{BIBLIOGRAFIA}

1. Ferenczy A. «Cervical Intraepithelial Neoplasia». Pathology of the female genital tract. Edited by Ancel Blaustein y Springer, VerlagNew York Inc. 1982.

2. Sadeghi SB et al. «Prevalence of cervical intraepithelial neoplasia in sexually active teenagers and young adults». Am. J. Obstet. Gynecol. 1984; 148: 7.

3. Gray LA. «Dysplasia, carcinoma in situ and microinvasive carcinoma of the cervix uteri» Edited by Chales C. Thomas Publisher, 1984.

4. Stalf A. «Diagnóstico y tratamiento de la neoplasia cervical intraepithelial». Clínicas Obstétricas y Ginecológicas 1983; 26: 4.

5. Reid BL. «The causation of cervical cancer». Clinics in Obstetrics and Gynecology 1985; 12: 1.

6. Baird PJ. «The role of papilloma and other viruses on cervical causation». Clinics in Obstetrics and Gynecology 1985; 12: 1 .

7. McCance Let al. «Human papillomavirus types 6 and 16 in multifocal intraepithelial neoplasias of the female lower tract». Br. J. Obstet. Gynaecol 1985; 92: 11.

8. Bernstein $\mathrm{A}$ et al. «The relationship of dietary and serum vit $\mathrm{A}$ to the ocurrence of CIN». Am. J. Obstet. Gynecol. 1984; 148: 3.

9. Hellberg D et al. «Smoking as risk factor in CIN». Lancet 1983; 31: 8365.

10. Clarke ES et al. «Cervix dysplasia: association with sexual behavior, smoking and oral contraceptive use?» Am. J. Obstet. Gynecol. 1985; 151: 5 .

11. Guijon FB et al. «The association of sexually transmited diseases with cervical intraepithelial neoplasia: a case control study» Am. J. Obstet. Gynecol. 1985; 151: 2.

12. Anderson $\mathrm{MC}$ «The pathology of cervical cancer». Clinics in Obstetrics and Gynecology 1985; 12: 1.

13. Giacomini G et al. «CIN or not CIN». Acta Cytologica 1983; 27: 5 .

14. Roy $\mathrm{M}$ et al. «Papilomavirus humano y las lesiones cervicales». Clínicas Obstétricas y Ginecológicas 1983; 26: 4.

15. Levine RU et al. «Cervical papillomavirus and cervical intraepithelial neoplasia: a study of male sexual partners». Obstet. Gynecol. 1984; 64: 1.

16. Singer $\mathrm{A}$ et al. «The wart virus and genital neoplasia; a causal or causal association». Br. J. Obstet. Gynaecol 1985; 92: 11.

17. Syrjanen $\mathrm{K}$ et al. «Natural history of cervical human paillomavirus infections based on prospective follow up». Br. J. Obstet. Gynaecol 1985; 92: 11 .

18. Reid R et al. «Genital warts and cervical cancer: VI. Relationship between aneuploid and plyploid cervical lesions». Am. J. Obstet. Gynecol. 1984; 150: 2.

19. Mazor MT «The coilocite and cervical intraepithelial neoplasia: time trend analysis of a recent decade». Am. J. Obstet. Gynecol. 1985; 150: 4.

20. Bamford PN et al. «The natural history of cervical intraepithelial neoplasia as determined by citology and colposcopic biopsy" Acta Cytologica 1983; 27: 5 .

21. Meisels A. «Problems in cytological screening from condylomatous lesions of the cervix». Clinics in Obstetrics and Gynecology 1985; 12: 1 .
22. Hudson E. «The prevention of cervical cancer: the place of the cytological smear test». Clinics in Obstetrics and Gynecology 1985; 12: 1.

23. Livolsi VA. «Cytologic screening intervals». Am. J. Obstet. Gynecol. 1984; 148: 6 .

24. Wright VC et al. «Age at beginning coitus vs. chronological age as a basis for Papanicolau screening». Am. J. Obstet. Gynecol. 1984; 149 : 8.

25. Fuji $\mathrm{F}$ et al. «Human papillomavirus infection and cervical intraepithelial neoplasia: Hystopathology and DNA content». Obstet. Gynecol. 1984; 63: 1.

26. Lavecchia C. et al. «Pap smear and the risk of cervical neoplasia: quantitative estimates from a case control study». Lancet 1984; 32: 8406.

27. Jordan JA. «Colposcopy in the diagnosis of cervical cancer and precancer», Clinics in Obstetrics and Gynecology 1985; 12: 1.

28. Walker PG et al. «Colposcopy in the diagnosis of papillomavirus infection of the uterine cervix». Br. J. Obstet. Gynaecol 1983; 90: 11.

29. Burke L \& Mathews BE. «Colposcopy in clinical practice». Edited by F.A. Davis Company-Philadelphia, 1977.

30. Hamou J et al. «Mycrohysteroscopy: a new approach to the diagnosis of cervical intraepithelial neoplasia». Obstet. Gynecol. 1984; 63: 4.

31. Singer A et al. «The treatment of CIN: conservative methods». Clinics in Obstetrics and Gynecology 1985; 12: 1.

32. Araf $\mathrm{HM}$ et al. "Cryosurgical treatment of cervical intraepithelial neoplasia». Am. J. Obstet. Gynecol. 1984; 150: 7.

33. Woodman $\mathbf{C B}$ et al. «The management of cervical intraepithelial neoplasia by coagulation electrodiathermy». Br. J Obstet. Gynaecol. 1985; 92: 7 .

34. Duncan ID. «El coagulador por frío en el tratamiento de la neoplasia intraepitelial cervical». Clínicas Obstétricas y Ginecológicas 1983; 26: 4 .

35. Baggish MS. «Tratamiento de la neoplasia cervical intraepitelial mediante el láser de $\mathrm{CO} 2 »$. Clínicas Obstétricas y Ginecológicas $1983 ; 26: 4$

36. Jordan JA et al. «The treatment of cervical intraepithelial neoplasia by laser vaporization». Br. J. Obstet. Gynaecol. 1985; 92: 4.

37. Sharp $\mathrm{F}$ et al. «The treatment of $\mathrm{CIN}$ : cone biopsy and hysterectomy». Clinics in Obstetrics and Gynecology 1985; 12: 1.

38. Jones III HW. «La conización en el tratamiento de la neoplasia cervical intraepitelial». Clínicas Obstétricas y Ginecológicas 1985; 12: 1 .

39. Trimbos JB et al. «Reliability of cytological follow-up after conization: a comparison of three surgical techniques». Br. J. Obstet. Gynaecol. 1983; 90: 7

40. Luesley DM et al. "Complications of cone biopsy related to the dimensions of the cone and the influence of prior colposcopic assesment». Br. J. Obstet. Gynaecol. 1985; 92: 2.

41. Fetherson W. «La citología falso negativa y el cáncer invasor del cérvix». Clínicas Obstétricas y Ginecológicas 1983; 26: 4.

42. Zuher MN. «Exfoliative cytopathology». Edited by Little Brown and Company Inc., 1970.

43. Carrera JM y cols. «Tratado y atlas de colposcopia». Editado por Salvat Editores, 1984. 